\title{
Integrated Analysis of Diffrentially Expressed Genes and Epigenetics Biomarkers in HCV-cirrhosis
}

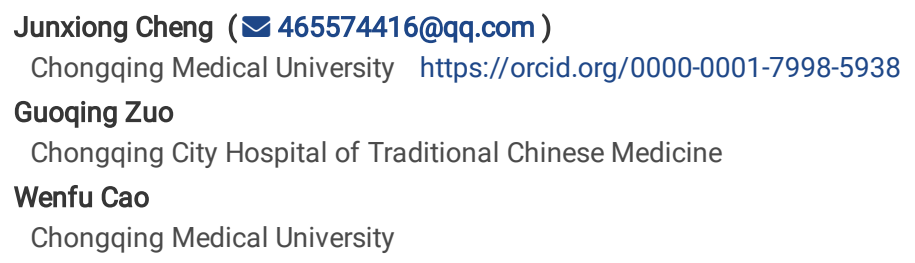

\section{Research}

Keywords: cirrhosis, DNA methylation, ISG15, TRIM22, IFI27, IFI16

Posted Date: June 23rd, 2021

DOI: https://doi.org/10.21203/rs.3.rs-626585/v1

License: () (i) This work is licensed under a Creative Commons Attribution 4.0 International License. Read Full License 


\section{Abstract}

Purpose

To identify the key genes and epigenetics biomarkers in HCV-cirrhosis based on informatics analysis of 4 GEO datasets.

Methods

After downloaded GEO datasets from NCBI, two GEO datasets (GSE6764 and GSE14323) were used to screen for the differentially expressed genes (DEGs) by limma package in R. Then DEGs were applied for Gene Ontology (GO) enrichment and Kyoto Encyclopedia of Genes and Genomes (KEGG) pathway enrichment analysis by clusterProfiler package in R. Protein-protein interaction network was constructed by cytoscape to identify the hub genes of HCVcirrhosis. DNA methylation dataset GSE60753 was analyzed by ChAMP package in R to identify the differentially methylated genes (DMGs). Cross-analysis of DEGs and DMGs were performed to identify the genes differentially expression and methylation, and further more indicated the methylation of them.

Results

357 DEGs and 8830 DMGs were identified in HCV-cirrhosis. Functional analysis of DEGs obtained pathways that may involved in the pathgenesis of HCVcirrhosis, including focal adhesion, influenza A, ECM-receptor interaction, protein digestion and absorption, etc. Cross-analysis of DEGs and DMGs identified 212 genes that changed in mRNA level and methylation status, and most of them were methylated in genebodies, but not CpG island. PPI construction in cytoscape revealed 25 hub genes in GEGs, and 5 of which were further analyzed for their probability as markers of HCV-cirrhosis by ROC curve and validation in another GEO dataset.

Conclusions

Our study identified the key genes in HCV-cirrhosis patient, which may provide new approach for clarifying the mechanism and new therapy of HCV-cirrhosis.

\section{Background}

Cirrhosis is a chronic liver desease characterized by esophageal varices, ascites, high portal pressure, etc, which caused by the formation of fibrous septae and nodules, collapse of liver structures[1]. Cirrhosis was usually induced by virus infection, alcoholic abuse, immune dysfuction, and biliary desease, which would lead to liver injury [2]. Among these etiologies, infection of Hepatitis $\mathrm{C}$ virus (HCV) plays an nonnegligible role in cirrhosis, expecially under the condition that the epidemic of Hepatitis B virus is getting well controll in China[3]. However, it is still not easy to prevent Hepatitis C progress into cirrhosis, especially caused by type $1 \mathrm{HCV}$ [4]. Therefore, it is necessary to identify the key genes of HCV-cirrhosis and to prepare for appropriate treatment.

DNA methylation is a basic biology process that plays an important role in cellular process and organ's function [5]. At present, mainstream understanding of methylation is that hyper methylation of DNA lead to downregulaiton or inhibition of gene expression [6], although there are still other findings against it [7]. Studies found that liver fibrosis are usually accompanied by methylation change in global level or particular genes [8-10]. However, the mechanisms that gene regulated by DNA methylation in cirrhosis still remains unclear.

Bioinformatics is an emerging and rapidly developing subject, which relies on the progress of sequencing technology and information technology to analyze big data [11], and have obtained many interesting research results. Bioinformatics analysis of the high-throughput sequencing data may provide new clue for the mechanism of HCV-cirrhosis. Therefore, it is useful to conduct an analysis combine gene expression and DNA methylation in HCV-cirrhosis.

In present study, 2 gene expression profiles GSE6764 and GSE14323, which from Gene Expression Omnibus (GEO) database were used to identify the differentially expressed genes. Meanwhile,a methylation profile GSE60753 was used to identify the differentially methylated CpG sites and genes. Moreover, construction of protein-protein interaction network of these DEGs revealed the potential associations between them. Finally, key genes validation were carried in GSE36411. These results may provide a new sight into the molecular mechanism and the relationship between DNA methylation and gene expression in HCV-cirrhosis.

\section{Materials And Methods Access of GSE datasets}

"HCV" and "cirrhosis" or "liver fibrosis" were used for retrieving in the GEO database, with a inclusion criteria: the dataset has at least two groups,including control and patient with HCV-cirrhosis, and sample size $>20$. Two expression profiling datasets( GSE6764, GSE14323) were included (Table.1). The data of GSE6764 was produced by GPL570([HG-U133_Plus_2] Affymetrix Human Genome U133 Plus 2.0 Array), with 13 HCV-Cirrhosis and 10 normal samples; and data of GSE14323 produced by GPL96([HG-U133A] Affymetrix Human Genome U133A Array), with 41 HCV-Cirrhosis and 19 normal samples.

Table.1 Dataset used for analysis in present study 


\begin{tabular}{|llll|}
\hline GEO ID & Platform & Control & HCV-Cirrhosis \\
\hline GSE14323 & GPL571 & 19 & 41 \\
\hline GSE6764 & GPL570 & 10 & 13 \\
\hline GSE60753 & GPL13534 & 33 & 38 \\
\hline GSE36411 & GPL10558 & 21 & 21 \\
\hline
\end{tabular}

\section{Identification of differentially expressed genes}

R packages "limma" and "preprocessCore" were used to perform background correction and quantile normalization respectively. Then, each probe names were converted into gene symbols according to GPL570 and GPL96 annotation files, respectively. The R package "limma" in R (v4.0.3) was further used to identify differentially expressed genes (DEGs) between patients with HCV-cirrhosis and control. False discovery rat (FDR) $<0.05$, adjusted $p$ value $<0.05$ and $\mid$ log2FCl 1 were considerd as the cutoff values for DEGs screening.

\section{Functional enrichment analysis of DEGs}

With the help of R package clusterProfiler, gene ontology (GO) and the KEGG signaling pathway enrichment analysis were performed on R by submit DEGs obtained in the previous step, pathways or processes with $p<0.05$ and gene number $\geq 5$ were recognized and regarded as the key pathways and processes.

\section{Methylation analysis of cirrhosis}

The GEO dataset GSE60753 contains DNA methylation profile of liver was downloaded and then their methylation status were analyzed by R package ChAMP. After quality control, 2 samples (GSM1487212 and GSM1487117) were discarded (Table.1). Thus, 34 control patients and 39 patients with HCV-cirrhosis were included.

The differentially methylated probe (DMP) was defined as cytosine-phosphate-guanine (CpG) sites with $\mid$ deltaBeta| $\geq 0.1$ and Adj.P.Val $<0.05$.Then, the genes displayed at least one DMP were identified as differential methylation genes (DMGs).

\section{Cross-analysis of DEGs and DMGs}

In order to know the affection of DNA methylation on gene expression in HCV-cirrhosis, all DEGs with methylation changement were identified, and the region of methylation in these genes were analysed.

\section{Construction of protein-protein interaction network}

All DEGs were submitted to STRING (http://string-db.org), and 303 DEGs were filterd into the PPI network, while 50 DEGs did not fall into the PPI network. After modified in cytoscape v3.6.0, a total of 303 nodes and 1932 edges displayed. Then, maximal clique

centrality method (MCC) in the plugin cytoHubba was used to get the hub-genes in these DEGs, the score $>1$ * $10^{\wedge} 11$ was considered significant and these genes were considered hub genes in HCV - cirrhosis.

\section{ROC curve analysis of methylated hub genes}

The whole methylation status of 25 hub genes were analysed by the DMP identified before, and only 14 hub genes accompanied with DNA methylation change, indeed hypermethylation. Then,the expression of these genes in GSE14323 dataset were applied to receiver-operator characteristic (ROC) curve analysis, $\mathrm{p}<0.05$ was considered statistically different, and these genes were considered key genes in HCV - cirrhosis.

\section{Validation of key genes}

In order to make this study convincible, another GEO dateset GSE36411, which contains HCV-cirrhosis patient and normal patients, was used to validate the hub genes expression. The expression of 5 genes between control group and HCV-cirrhosis group were analyzed by student's t-test in GraphPad Prism 8.0, and $p<0.05$ was considered statistically different.

\section{Results}

\section{Identification of DEGs in HCV-Cirrhosis}

Performed as described above, 856 genes upregulated and 273 genes downregulated in GSE6764 dataset, while 737 genes upregulated and 10 genes downregulated in GSE14323 dataset. Then, DEGs identified in each dataset were visualized in heatmaps and volcano plots (Fig. 1, Fig. 2). A total of 357 genes which were differentially expressed in HCV-Cirrhosis and normal samples were identified as DEGs. Totally, there are 350 up-regulated genes and 7 downregulated DEGs shared by both GSE6764 dataset and GSE14323 dataset, as listed in Table 2.

Table. 2 A panel of DEGs identified in the two datasets. 


\section{Functional enrichment analysis of DEGs}

In order to exhibit the gene function of all DEGs, GO and KEGG analyses were performed: the top 8 significant GO and KEGG pathways are shown in Figure.3. The GO analysis including 4 part: molecular function (MF), biological process (BP), and cellular component (CC), and extracellular matrix structural constituent, extracellular matrix organization, collagen-containing extracellular matrix and focal adhesion are the most significant function or pathway in MF, BP, CC and KEGG pathways. Interestingly, response to virus is also a significant function in BP; influenza A is a significant pathway in KEGG pathway.

\section{Identifying of differentially methylated genes}

As DNA methylation regulates gene expression in a complicated way, $25125 \mathrm{CpG}$ site were identified as differentially methylated probes (DMPs) and 8,830 genes were identified as differentially methylated genes (3831 genes have only one DMP, the other have at least 2 DMPs), the top 1000 DMGs were shown in Fig. 4.

Cross-analysis of DMGs and DEGs identified 212 genes ( supplementary table 1), 131 genes upregulated and hypermethylated, 77 genes upregulated and hypomethylated, 2 genes downregulated and hypermethylated, 2 genes downregulated and hypomethylated. At the same time, almost half of the DMPs were found to be in the gene body (12777), while only $3493 \mathrm{CpG}$ methylation occured in island.

\section{Protein-protein interaction construction and hub genes identification}

To better understand which of the shared DEGs were most likely to be the key genes in the development of HCV-cirrhosis, a PPI network for 357 DEGs was constructed (supplementary Fig. 1), 54 genes were not included in this PPI network. According to the MCC method in CytoHubba, 25 genes significant were as follows: OASL, OAS1, OAS2, MX1, OAS3, ISG15, IFIT1, TRIM22, MX2, IFI35, IFI6, STAT1, IFIH1, IFI44L, IFI44, BST2, IFI27, CXCL10, HERC6, RTP4, HERC5, DDX60, ISG20, IFI16, SP110 (Fig. 5). These 25 genes were deemed to be the hub genes and were most likely to be essential for fibrogenesis in HCV patient.

\section{Methylation status of hub genes}

Then the methylation changement among the 25 hub genes were analyzed. 11 genes, include OASL, OAS3, IFI35, IFI6, IFIH1,IFI44, CXCL10,HERC6, RTP4, HERC5, SP110, displayed no significant methylation change, while the other 14 hub genes displayed a significant change in at leaset one CpG site (supplementary table 2). Interestingly, no methylation change happens in $\mathrm{CpG}$ island of these hub genes.

ROC curve analysis

The performance of the 14 differentially methylated hub genes as diagnostic biomarkers was examined by ROC curves. Results indicated that, ISG15, TRIM22, IFI44L, IFI27 and IFI16 are potential markers with AUC value $>0.99$, and $P$ value $<0.0001$. (Fig. 6).

\section{Validation of hub genes}

ISG15, TRIM22, IFI44L, IFI27 and IFI16 in GSE36411 were analyzed to comfirm the expression of the methylated hub genes. As shown in Fig. 7, ISG15, TRIM22, FI27 and IFI16 significantly increased in HCV-cirrhosis patient.

\section{Disscussion}

As the progression of cirrhosis is not fully understood, the treatment is still limited. In present study, cross analysis of HCV-cirrhosis was carried out. DEGs were fitered, hub genes were predicted by STRING and cytoscape,GO and KEGG analysis of DEGs were performed as well. The methylation of hub genes were analyzed. All of these should provide a comprehensive understanding of HCV-cirrhosis.

A total of 357 genes were identified as DEGs, including fibrosis initiation factor TGF- $\beta$, PDGFA and PDGFD, which were critical to the activation of hepatic stellate cells [12] [13]. Meanwhile, the collogen deposition were increased, including COL1A1, COL1A2, COL4A1, COL4A2, COL4A3,etc. Other important genes, such as STAT1, CXCL family that correlate to cirrhosis increased as well.

GO enrich analysis of DEGs demonstrated that molecular function mainly enriched in extracellular matrix structural constituent, glycosaminoglycan binding, receptor ligand activity; and that biological function mainly enriched in extracellular matrix organization, extracellular structure organization, response to virus, defense response to virus, regulation of cell - cell adhesion; and that cellular component mainly enriched in collagen - containing extracellular matrix endoplasmic reticulum lumen, external side of plasma membrane. It is explicit that extracellular matrix deposition is the basic pathological change in cirrhosis. Meanwhile, the response and defense response to virus also enriched in DEGs. Previous research has shown that the response to HCV result in chronic inflammation and become a vital activator for myofibroblast transdifferentiation [14]. HCV infection not only destroy the hepatocytes, but also induces the expression of TGF- $\beta$ family [15]. So, antiviral is the principal therapy for HCV-cirrhosis [16]. Study also emphasized that cirrhosis could be reversible when the etiology is removed [17]. These indicated the importance of TGF-ßin cirrhosis.

KEGG analysis of common DEGs reveals the main pathyways are focal adhesion, influenza A, ECM - receptor interaction, protein digestion and absorption. Focal adhesion kinase, member of tyrosine kinase superfamily,was found to regulate the activation of HSC and liver fibrosis [18]. PI3K are member of focal 
adhesion pathway, and PI3K/AKT/mTOR induced autophage activate HSCs, and thus lead to fibrosis [19]. Meanwhile, the identified DEGs in AGE-RAGE signaling pathway in diabetic complications, including TGF- $\beta 1$, BCL2, STAT1, MMP2 were found to link to cirrhosis[20]. Interestingly, VEGFC was found to benefit cirrhotic rats with hepatic encephalopathy [21]. However, the detailed mechanism still need further study.

DNA methylation is a key event in cellular and molecular biological processes. Although past studies have shown a reduced mehtylation level in $\mathrm{CCl}_{4}$ induced liver fibrosis [10], there are still controversial findings that DNA methylation increased in particular genes which were upregulated in fibrosis model [22] Confusingly, a longer $\mathrm{CCl} 4$ exposure did not significantly change DNA methylation status [23]. On the other hand, methylation in promoters of several genes including P14, P15, P73, MGMT were found to be increased in liver disease including cirrhosis [24]. In a word, the diversity of DNA methylation status in cirrhosis is undeniable. In fact, gene expression may not negatively correlated with DNA methylation level, because their methylation may increase the binding affinity of their corresponding transcription factors. [25]. Although methylation at CpG island (CGI) of transcription start sites (TSS) was know to repress gene expression,while it is still unkown in non-CGI TSS [26]. Our results indicated that not all DEGs were hyper- or hypo-methylated. of the 25 hub genes, 11 genes showed no mehtylation change, and 6 gene hyper-methylated in only $1 \mathrm{CpG}$ site, while the others in more than 1 site. Thus, it is difficult to judge the effection of DNA methylation on expression of gene which were methylated at different site. More detailed studies are needed to clarify the specific mechanism.

We screened out 25 genes as hub genes, including OAS family, MX family, IFI family, HERC family and others. OAS1, MX1 polymorphisms was found to associated with the severity of liver disease in HIV and HCV co-infected patients [27] [28]. Morever, MX1 was up-regulated in the activated HSCs [29]. Thus, we might infer that, MX1 promotes HSC activation and is related to the prognosis of HCV-cirrhosis, althouth further study is needed to clarify this. In addition, OAS1 and IFI44 expression were found to be increased in patients with systemic sclerosis-related interstitial lung disease, while there is no study focused on their function in cirrhosis. Of the identified 5 biomarkers, IFI27 and ISG15 were shown to increased significantly in non-sustained virological response (SVR) patients than in SVR patients [30]; while TRIM22, IFI44L, and IFI16 is still lack of investigation. Thus, we infer that OAS1 and MX1 may be the epigenetics biomarkers of HCV-cirrhosis. However, more detailed work is needed to clarify their function in HCV-cirrhosis.

\section{Conclusion}

We identified the DEGs in cirrhosis, and the related pathway, including focal adhesion, influenza A, protein digestion and absorbtion, which may be involved in HCV-cirrhosis. Furthermore, we preliminarily discussed the effection of DNA methylation on gene expression in cirrhosis, which still need further study to explore. And proposed that OAS1 and MX1 may be the epigenetics biomarkers of HCV-cirrhosis.

\section{Declarations}

\section{Acknowledgements}

Not applicable.

\section{Author contributions}

Wenfu Cao and Guoqing Zuo conceived and designed the study. Junxiong Cheng and Wenfu cao performed the data analysis. Junxiong Cheng wrote the manuscript. All authors are responsible for reviewing data. All authors read and approved the final manuscript.

\section{Funding}

This work was funded by the National Natural Science Foundation of China (Grant No.: 81573860).

\section{Availability of data and materials}

Data available within the article or its supplementary materials.

\section{Declarations}

\section{Ethics approval and consent to participate}

Not applicable.

\section{Consent for publication}

Written informed consent for publication was obtained from all participants.

\section{Competing interests}

None.

\section{Author details}

${ }^{1}$ College of Traditional Chinese Medicine, Chongqing Medical University, Chongqing Key Laboratory of Traditional Chinese Medicine for Prevention and Cure of Metabolic Diseases, Chongqing 400016, P.R. China. ${ }^{2}$ Department of Gastroenterology, Chongqing Hospital of Traditional Chinese Medicine, Chongqing 400021, P.R. China. 


\section{References}

1. Parola M, Pinzani M. Liver fibrosis: Pathophysiology, pathogenetic targets and clinical issues. Mol Aspects Med. 2019;65:37-55.

2. Zhang CY, Yuan WG, He P, et al. Liver fibrosis and hepatic stellate cells: Etiology, pathological hallmarks and therapeutic targets. World J Gastroenterol. 2016;22(48):10512-22.

3. Scaglione,Steven; Kliethermes SC, Shoham G, Durazo D, Luke R, Volk A, Michael L. The Epidemiology of Cirrhosis in the United States: A Population-based Study. J Clin Gastroenterol. 2015; 49 (8): 690-696.

4. Kohli A, Shaffer A, Sherman A, et al. Treatment of hepatitis C: a systematic review. JAMA. 2014;312(6):631-40.

5. Moore LD, Le T, Fan G. DNA methylation and its basic function. Neuropsychopharmacology. 2013;38(1):23-38.

6. Borowa-Mazgaj B, de Conti A, Tryndyak V, et al. Gene Expression and DNA Methylation Alterations in the Glycine N-Methyltransferase Gene in Diet-Induced Nonalcoholic Fatty Liver Disease-Associated Carcinogenesis. Toxicol Sci. 2019;170(2):273-82.

7. Ding Z, Qian Y-B, Zhu L-X, et al. Promoter methylation and mRNA expression of DKK-3 and WIF-1 in hepatocellular carcinoma. World J Gastroenterol. 2009;15(21):2595.

8. Wilson CL, Mann DA, Borthwick LA. Epigenetic reprogramming in liver fibrosis and cancer. Adv Drug Deliv Rev. 2017;121:124-32.

9. Bian EB, Zhao B, Huang C, et al. New advances of DNA methylation in liver fibrosis, with special emphasis on the crosstalk between microRNAs and DNA methylation machinery. Cell Signal. 2013;25(9):1837-44.

10. Komatsu Y, Waku T, Iwasaki N, et al. Global analysis of DNA methylation in early-stage liver fibrosis. BMC Med Genomics. 2012;5:5.

11. Gauthier J, Vincent AT, Charette SJ, et al. A brief history of bioinformatics. Brief Bioinform. 2019;20(6):1981-96.

12. Dewidar M, Dooley, et al. TGF- $\beta$ in Hepatic Stellate Cell Activation and Liver Fibrogenesis-Updated 2019. Cells. 2019;8(11):1419.

13. Borkham-Kamphorst E, Meurer SK, Van de Leur E, et al. PDGF-D signaling in portal myofibroblasts and hepatic stellate cells proves identical to PDGF-B via both PDGF receptor type alpha and beta. Cell Signal. 2015;27(7):1305-14.

14. Irshad M, Gupta P, Irshad K. Immunopathogenesis of Liver Injury During Hepatitis C Virus Infection. Viral Immunol. 2019;32(3):112-20.

15. Chida T, Ito $\mathrm{M}$, Nakashima $\mathrm{K}$, et al. Critical role of $\mathrm{CREBH}$-mediated induction of transforming growth factor beta2 by hepatitis $\mathrm{C}$ virus infection in fibrogenic responses in hepatic stellate cells. Hepatology. 2017;66(5):1430-43.

16. Roehlen N, Crouchet E, Baumert TF. Liver Fibrosis: Mechanistic Concepts and Therapeutic Perspectives. Cells. 2020; 9 (4).

17. Sun M, Kisseleva T. Reversibility of liver fibrosis. Clin Res Hepatol Gastroenterol. 2015;39(Suppl 1):60-3.

18. Zhao XK, Yu L, Cheng ML, et al. Focal Adhesion Kinase Regulates Hepatic Stellate Cell Activation and Liver Fibrosis. Sci Rep. 2017;7(1):4032.

19. Wang H, Liu Y, Wang D, et al. The Upstream Pathway of mTOR-Mediated Autophagy in Liver Diseases. Cells. 2019; 8 (12).

20. Alberto Martí-Rodrigo FA, Ángela B, Moragrega, Francisco García-García, Pablo Martí-Rodrigo, Anabel Fernández-Iglesias, Jordi Gracia-Sancho, N, Apostolova JV, Esplugues, Ana Blas-García. Rilpivirine attenuates liver fibrosis through selective STAT1-mediated apoptosis in hepatic stellate cells. Gut. 2020; 69 (5): 920-932.

21. Hsu SJ, Zhang C, Jeong J, et al. Enhanced Meningeal Lymphatic Drainage Ameliorates Neuroinflammation and Hepatic Encephalopathy in Cirrhotic Rats. Gastroenterology. 2021; 160 (4): 1315-29 e13.

22. Wu P,Rui Huang Y-LX, Chao Wu. Protective effects of curcumin against liver fibrosis through modulating DNA methylation. Chin J Nat Med. 2016; 14 (4): 255-264.

23. Page A, Paoli P, Moran Salvador E, et al. Hepatic stellate cell transdifferentiation involves genome-wide remodeling of the DNA methylation landscape. J Hepatol. 2016;64(3):661-73.

24. Zekri Ael R, Nassar AA, El-Din El-Rouby MN, et al. Disease progression from chronic hepatitis $\mathrm{C}$ to cirrhosis and hepatocellular carcinoma is associated with increasing DNA promoter methylation. Asian Pac J Cancer Prev. 2014;14(11):6721-6.

25. Yin Y, Morgunova E, Jolma A, et al. Impact of cytosine methylation on DNA binding specificities of human transcription factors. Science. 2017 ; 356 (6337).

26. Jones PA. Functions of DNA methylation: islands, start sites, gene bodies and beyond. Nat Rev Genet. 2012;13(7):484-92.

27. Din BE, Anany NG, Dawood MA. RM, et al. Impact of OAS1 Exon 7 rs10774671 Genetic Variation on Liver Fibrosis Progression in Egyptian HCV Genotype 4 Patients. Viral Immunol. 2015;28(9):509-16.

28. Garcia-Alvarez M, Berenguer J, Jimenez-Sousa MA, et al. Mx1, OAS1 and OAS2 polymorphisms are associated with the severity of liver disease in HIV/HCV-coinfected patients: A cross-sectional study. Sci Rep. 2017;7:41516.

29. Yaogang Zhong,Xiu-Xuan. Sun PZ, Qin X, Chen W, Guo Y, Jia Z, Bian H, Li Z. Identification and localization of xylose-binding proteins as potential biomarkers for liver fibrosis/cirrhosis. Mol Biosyst. 2016; 12 (2): 598-605.

30. Domagalski K, Pawlowska M, Kozielewicz D, et al. The Impact of IL28B Genotype and Liver Fibrosis on the Hepatic Expression of IP10, IFI27, ISG15, and MX1 and Their Association with Treatment Outcomes in Patients with Chronic Hepatitis C. PLoS One. 2015;10(6):e0130899.

\section{Figures}


A

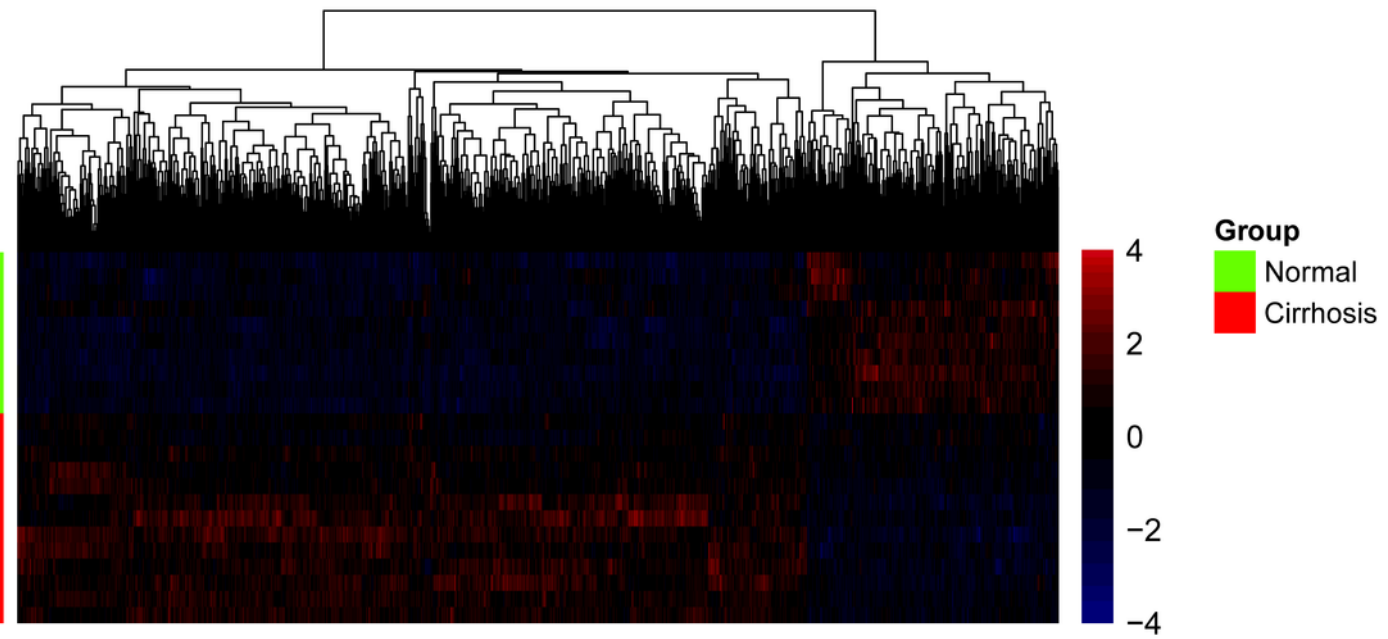

B

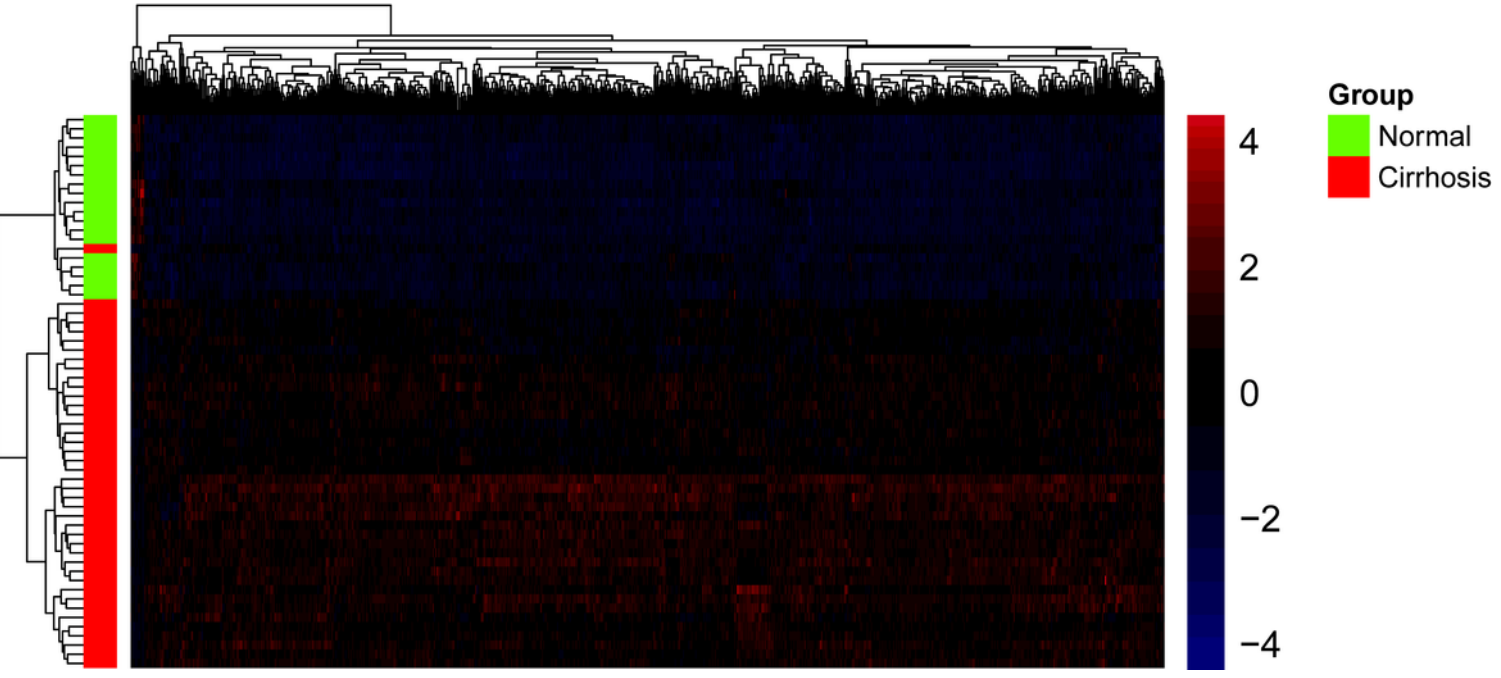

Figure 1

Heat map of DEGs. All differentially expressed genes in GSE6764 (A) and GSE14323 (B) are showed. 

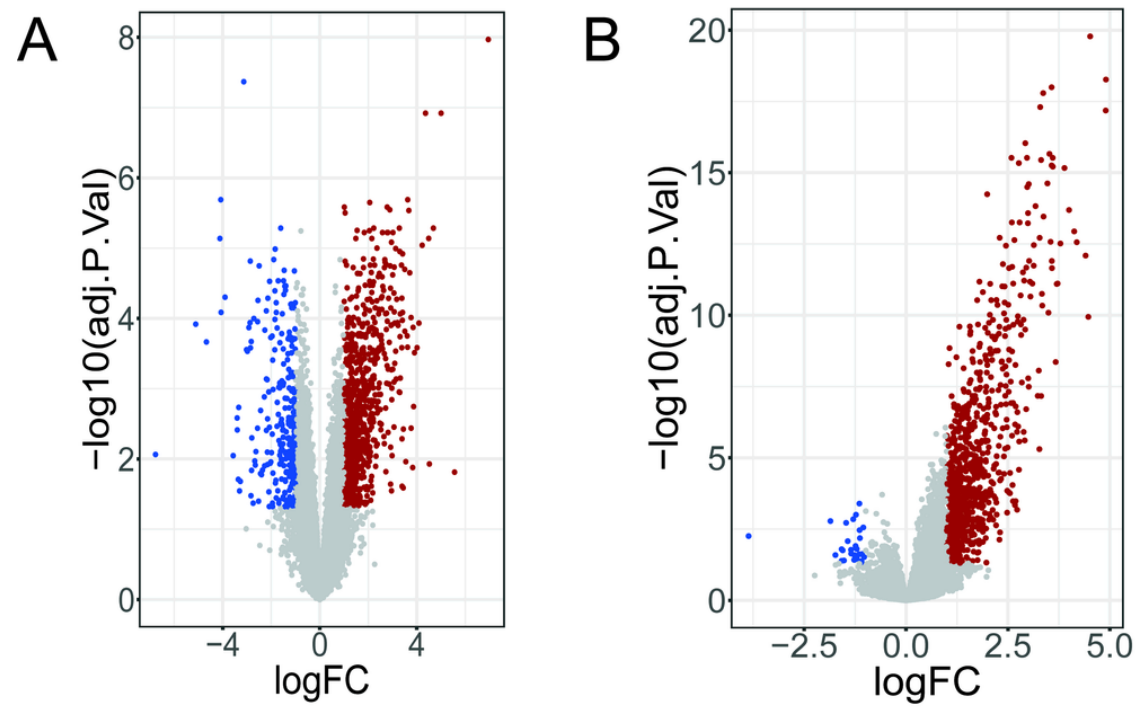

\section{Change \\ - Down \\ - Not sig \\ - Up}

C

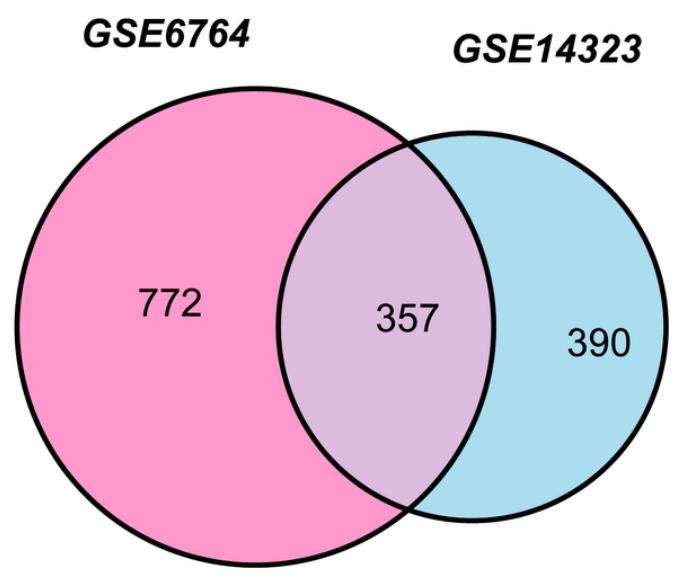

Figure 2

The volcano plots and Venn diagrams of DEGs. Each point in the volcano map represents a gene. Red represents up-regulated genes, blue represents downregulated genes and gray represents genes that are not significantly changed.A:GSE6764; B: GSE14323. 
A

extracellular matrix structural constituent

glycosaminoglycan bindin

platelet-derived growth factor binding

extracellular matrix structural constituent conferring tensile strength

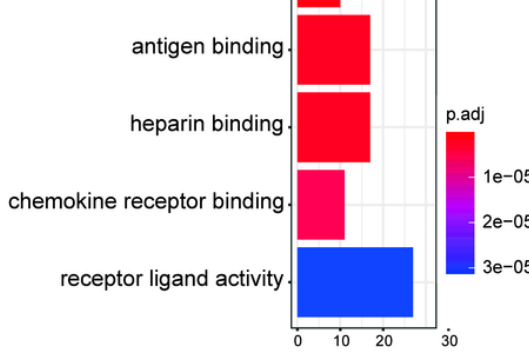

C

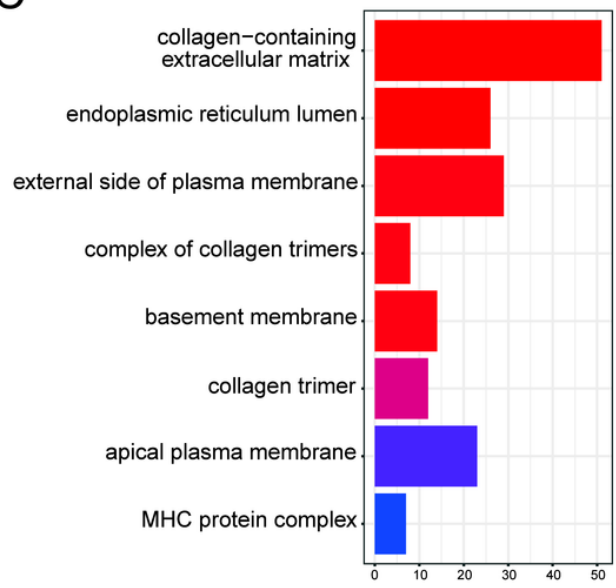

B

extracellular ma:rix organization

extracellular structure organization

ressponse to virus.

cell chemotaxis

defense ressponse to virus.

urogenital system development

05

leukoryte chemotaxis

regulation of ce:l-cell adhesion.

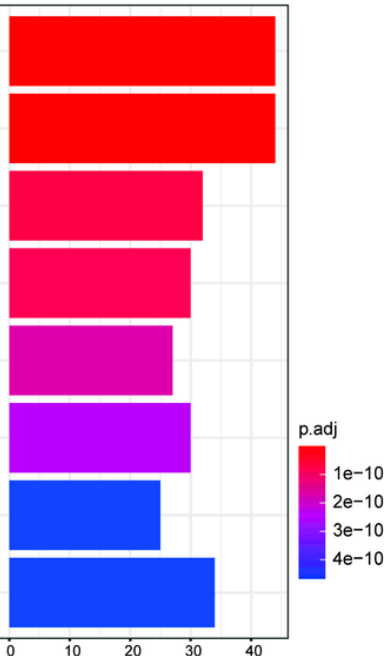

D

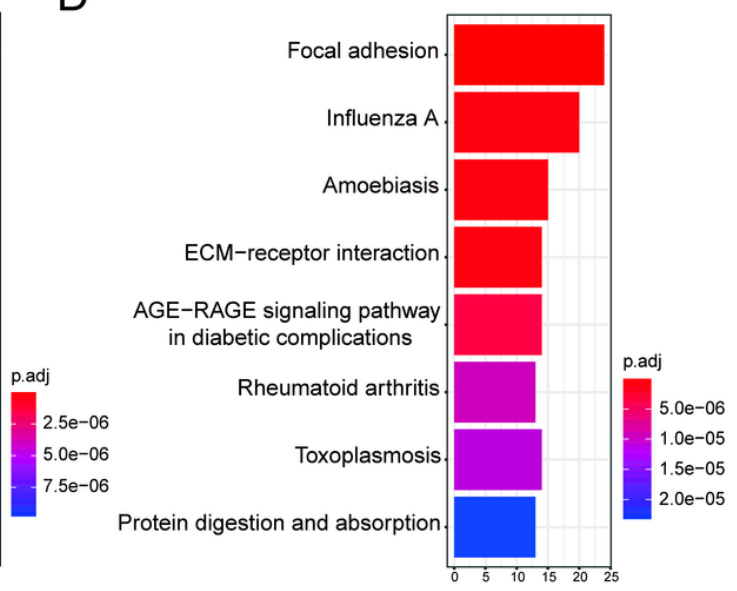

\section{Figure 3}

The top 8 significant enrichment GO terms and KEGG pathways of DEGs in HCV-Cirrhosis.A: Molecular function. B: Biological process. C: Cellular component. D: KEGG pathways. The $x$ axis shows numbers of each terms, the $y$ axis shows $G O$ terms or KEGG pathways and color shows the adjusted $p$ value. 


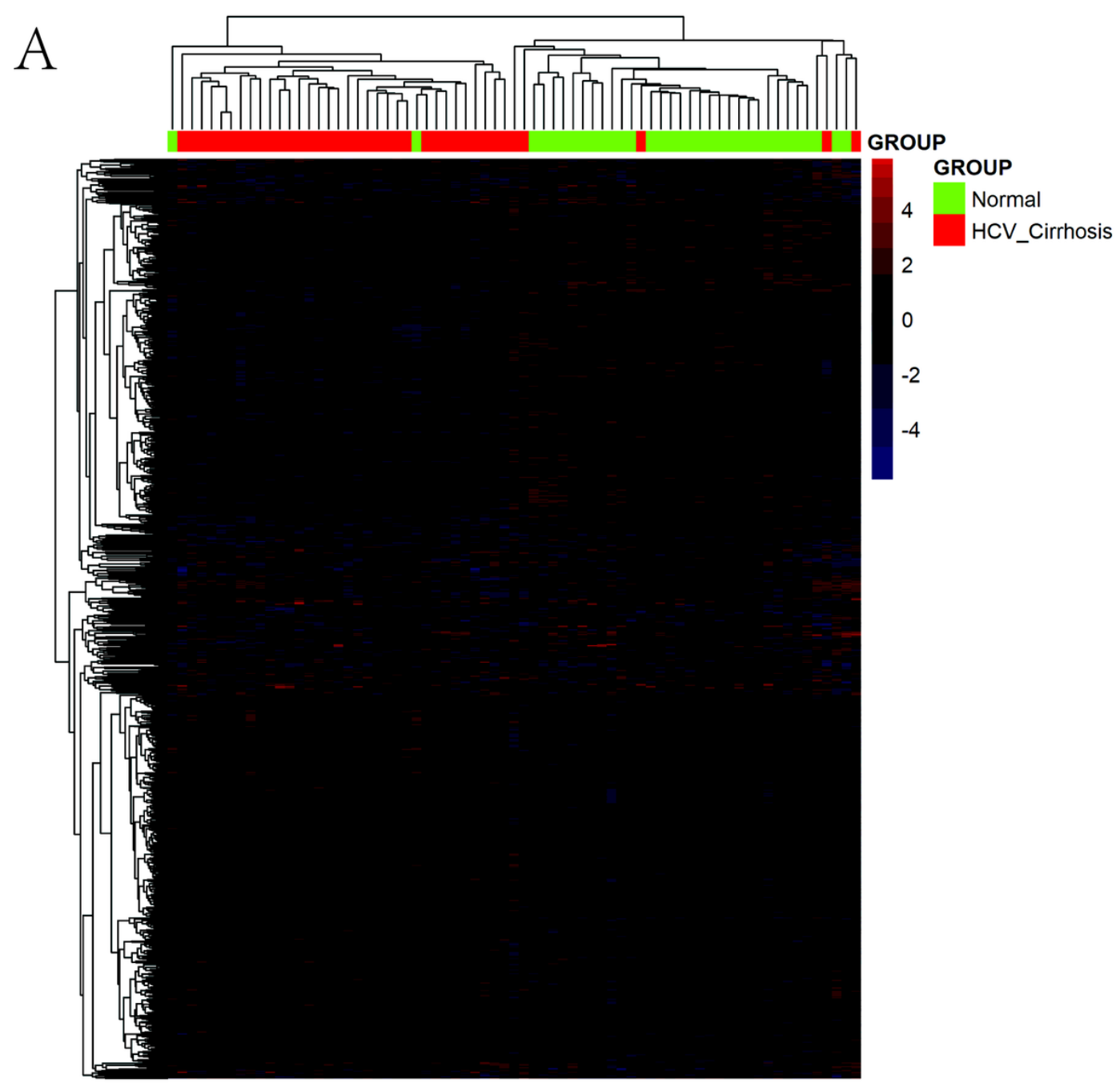

Figure 4

The DMGs of GSE60753. A: Heatmap of the top 1000 DMGs of GSE60753. B: The relationship between DEGs and DMGs. 


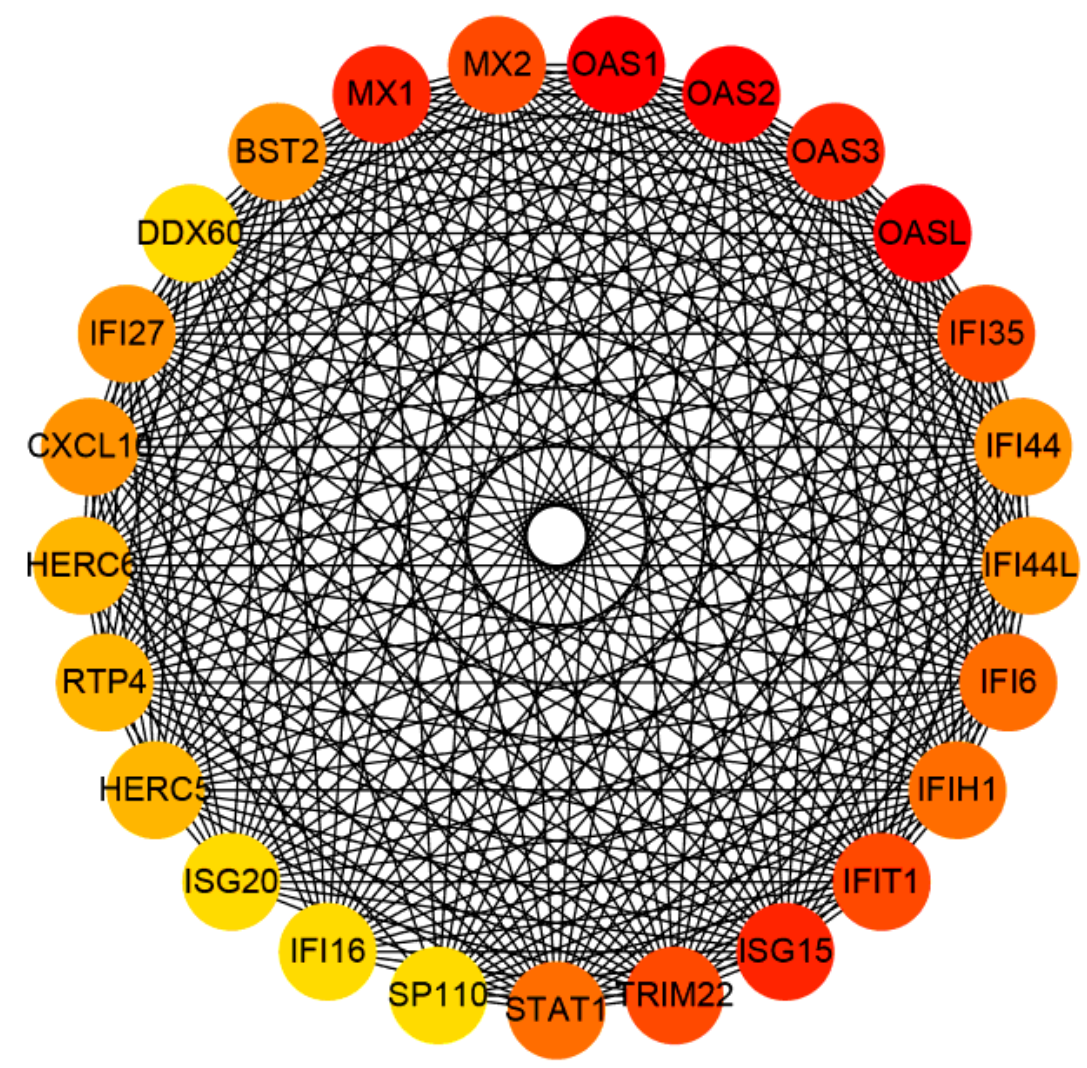

Figure 5

Top 25 hub genes network of HCV-cirrhosis. A redder color represent a high score in cytoscape by MCC method. 
A

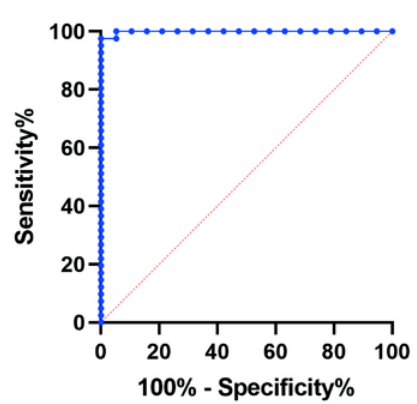

C

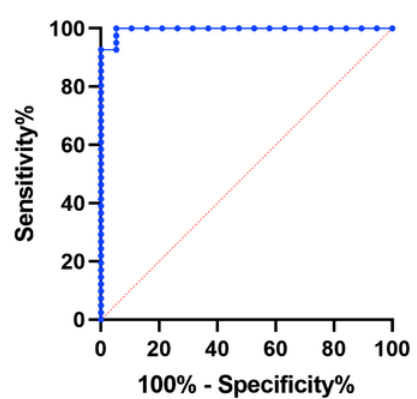

E

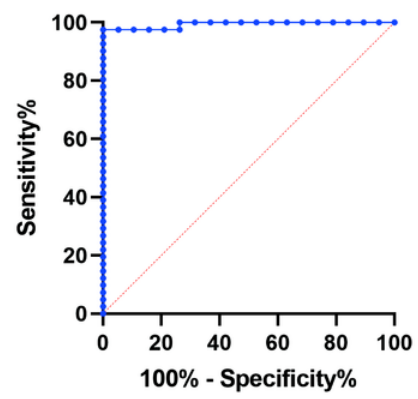

B

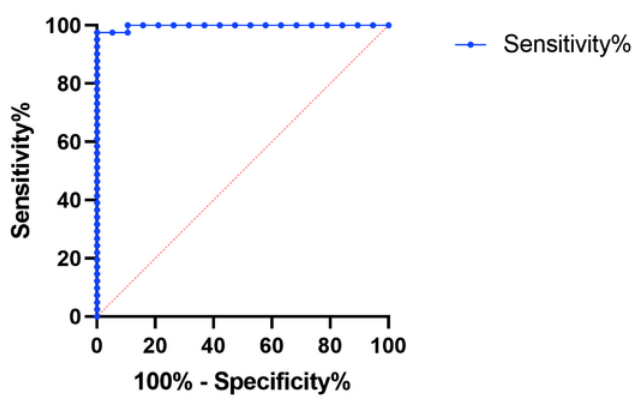

$\rightarrow$ Sensitivity $\%$

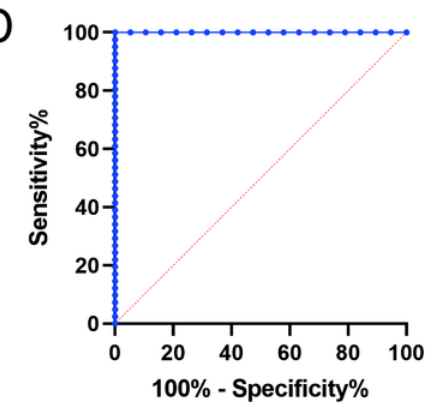

- Sensitivity\%

$\rightarrow$ Sensitivity $\%$

Figure 6

ROC curves of ISG15, TRIM22, IFI44L, IFI27 and IFI16 for HCV-cirrhosis diagnosis. A: ISG15,AUC=0.9987(0.9945-1.000, P value<0.0001); B:

TRIM22,AUC=0.9974 (0.9906-1.000, P value<0.0001); C: IFI44L, AUC=0.9961 (0.9868 - 1.000, P value<0.0001); D: IFI27, AUC=1 (1.000 - 1.000, P value<0.0001); E: IFI16, AUC= $0.9936(0.9794-1.000, P$ value<0.0001)

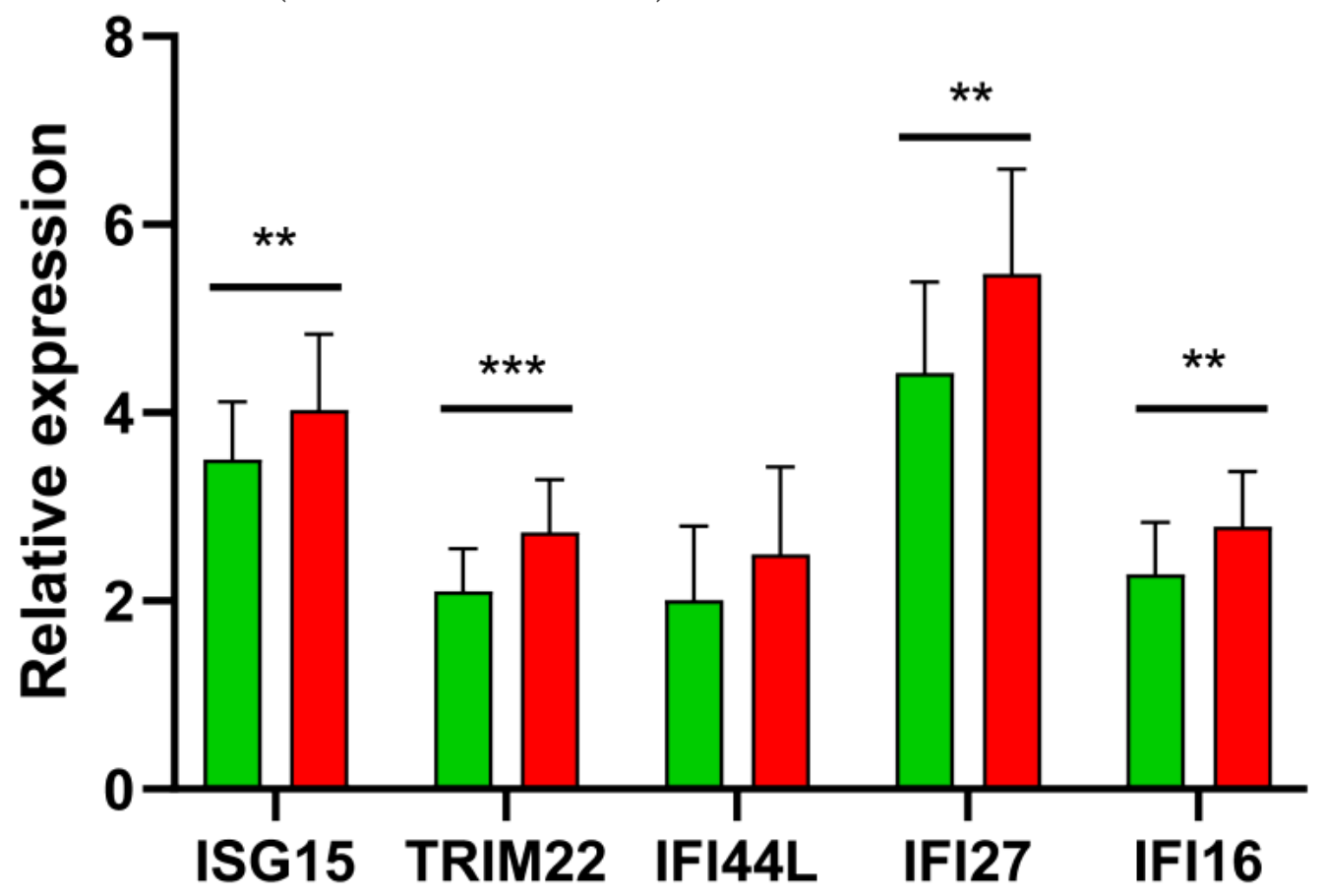


Figure 7

Validation ISG15, TRIM22, IFI44L, IFI27 and IFI16 in GSE36411. The expression differences of 5 hub genes between the normal group and HCV-fibrosis group were statistically significant except IFI44L. **p $<0.01,{ }^{\star \star \star} \mathrm{p}<0.001$.

\section{Supplementary Files}

This is a list of supplementary files associated with this preprint. Click to download.

- supplementaryfigure1.tif

- supplementarytable1.docx

- supplementarytable2.docx 